\title{
Precue effects in visual search: Data or resource limited?
}

\author{
RONALD A. KINCHLA, ZHE CHEN, and DENISE EVERT \\ Princeton University, Princeton, New Jersey
}

\begin{abstract}
Precuing an observer as to where a target is more likely to occur in a subsequent visual array can increase the detectability $\left(d^{\prime}\right)$ of a target at that location. This is often attributed to the observer's increased allocation of some limited cognitive resource ("attention") to the cued location. Two experiments are reported which are difficult to interpret in this way even though they involve similar cue effects. The first involves postcuing a location well after the array but before the observer responds, so that the cue can influence the response but not the observation. The second involves precuing, but with slow sequential presentation of array elements prior to the response, so the observer need not share any limited resource while processing each element in turn. Enhanced detectability similar to that produced with precues and simultaneous presentation of elements is shown to occur in each experiment. An alternative data-limited (rather than resource-limited) interpretation of these effects is provided by a mathematical model in which the observer integrates equally noisy or degraded internal representations of the array elements, but gives more weight to cued elements in selecting a response. Theoretical parameters of the model are shown to provide separate measures of both an observer's overall sensitivity and precue effects in cost-benefit analyses of cuing data.
\end{abstract}

In a typical visual search task, observers evaluate each of a series of multielement visual arrays for the presence of a target element, responding "yes" when they decide an array contains a target and "no" otherwise. Employing a distinction suggested by Norman and Bobrow (1975), one could describe theories of visual search as either "resource" or "data" limited. A resource-limited view is that an observer's principle problem is sharing some limited cognitive resource (e.g. "attention") among the simultaneously presented elements of an array. In contrast, the principle problem from a data-limited view is the noisy or degraded internal representations of array elements caused by their brief exposure, subsequent masking, or the need to respond rapidly before better representations can be formed. For example, the two theoretical views imply very different explanations of why target detectability diminishes as the number of elements in an array is increased, the so called set-size effect. The limited-resource explanation of this effect is that, in a parallel search, each element receives a progressively smaller share of the resource as the number of elements is increased (Rumelhart, 1970); in a serial search, it takes progressively longer to evaluate all the elements in the array, some of which may be lost from "iconic" memory before processing can occur (e.g., Estes \& Taylor, 1964; Treisman \& Gelade, 1980). In contrast, the data-limited explanation is that each additional element simply increases the risk of confusing the noisy representation of a nontarget with that of a target.

Correspondence should be directed to R. A. Kinchla, Department of Psychology, Green Hall, Princeton University, Princeton, NJ 085441010 (e-mail:kinchla@phoenix.princeton.edu).
Note that the preceding distinction is not the same as that between early and late selection theories. For example, a resource limitation can arise "early" in the sensory system, as a kind of filter or differential attenuator (e.g., Treisman, 1969), or "late" in processing, as a limit on the amount of information that can be searched or rehearsed in short-term memory (e.g., Deutsch \& Deutsch, 1963; Durican, 1980).

A critical test of whether a visual search task is data or resource limited is afforded by studies in which array elements are sometimes presented simultaneously and sometimes in slow succession. A limited-resource theory would predict much higher accuracy with successive presentation, since the entire resource can be successively allocated to each element in turn. However, a data-limited theory would predict similar levels of accuracy with either mode of presentation, as long as the factor limiting the quality of each internal representation (e.g., exposure duration) remained the same. Two such studies, one by Eriksen and Spencer (1969) and the other by Shiffrin and Gardner (1972), support the data-limited view, inasmuch as accuracy was the same for both modes of presentation. ' Furthermore, the identical set-size effects obtained by Eriksen and Spencer for each type of presentation are consistent with a "data-limited" mathematical model of visual search in which each array element adds the same amount of noise to an observer's overall impression of an array (Kinchla, 1969, 1974).

In this paper, we consider how the distinction between data and resource limitations relates to another aspect of visual search, the effect of indicating to an observer where a target is most likely to occur in a subsequent array. This precue effect is an increase in the observer's ability to de- 
tect a target in the precued location as measured by $d^{\prime}$, with reduced ability elsewhere. Since this occurs without eye movements, it is widely interpreted as reflecting a covert shift in "attention" induced by the precue (a limitedresource explanation). A representative precue effect obtained by Müller and Findlay (1987) is shown in Figure 1 . They used briefly presented, four-element arrays, of which a random two-thirds contained a single target element. Some arrays were preceded by a neutral cue $(\mathrm{N})$, which indicated that each of the four elements was equally likely to be the single target element. Other arrays were preceded by an arrow, indicating that one of the four elements was more likely to be a target than each of the other three. When a target was presented, it occurred $44 \%$ of the time in the location indicated by the arrow, a valid cue (V); otherwise it occurred equally often in each of the other three locations, an invalid cue (I). Target detectability $\left(d^{\prime}\right)$ following each type of cue (I, N, V) is presented in Figure 1 in the form of a "costbenefit analysis" (Posner \& Snyder, 1975). The three data points in Figure 1 are estimates of $d^{\prime}$ based on part of Müller and Findlay's (1987) data. (The solid line indicates corresponding theoretical values which will be discussed later in this paper.) The typical precue effect is evident in Figure 1. Relative to neutral cue trials, target detectability $\left(d^{\prime}\right)$ was enhanced when the arrow cue was valid, the "benefit," and diminished when it was invalid, the "cost." (While questions have been raised concerning certain aspects of this study, ${ }^{2}$ the precue effect evident in Figure 1 is typical of such studies.)

While Müller and Findlay (1987) acknowledge that their precue effects might be interpreted in terms of decision-making factors in a data-limited process, they conclude that the total pattern of data from their study supports a capacity-limited view of visual search. In this paper, we present two cuing experiments which are dif-

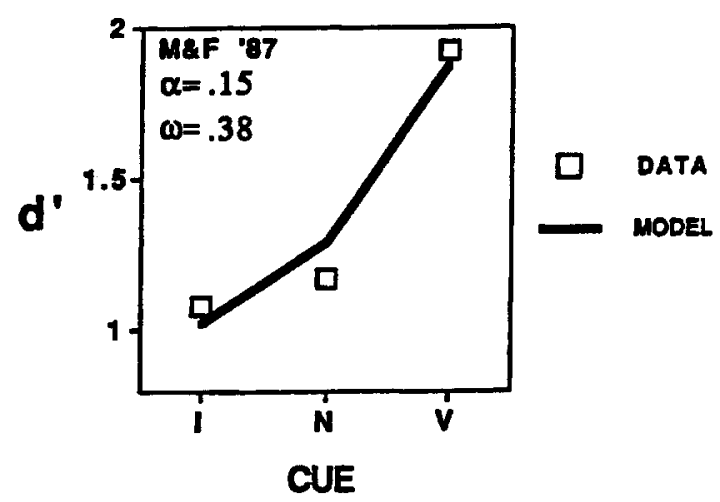

Figure 1. A cost-benefit analysis of data from a visual search study by Müller and Findlay (1987) involving briefly flashed four-element arrays. The data points indicate target detectability $\left(d^{\prime}\right)$ given an invalid (I), neutral (N), or valid (V) precue. The solid line indicates corresponding theoretical values based on a two-parameter form of the weighted integration model developed later in this paper: $\alpha$ is the variance (noise) of each elementary impression; $\omega$ is the weight assigned to the precued element, assuming each of the other three elements was assigned a weight of $(1-\omega) / 3$. ficult to interpret in terms of a resource-limited view but are susceptible to a data-limited interpretation. Experiment 1 involved postcuing the observer as to where a target was more likely after presentation of the array but before the response, so that the postcue could influence the decision-making process but would occur too late to influence the allocation of attention during the observation. Experiment 2 employed precues with slow, sequential presentation of briefly flashed array elements, so that observers could allocate their total attention to each element in turn, eliminating the advantage normally attributed to the precued element in a limited-resource view. In contrast, a data-limited view of this task, where cue effects arise in the decision process, would predict postcue effects in the first experiment similar to those with precues and, in the second experiment, precue effects comparable to those obtained with simultaneous presentation.

Before presenting the two experiments we will describe the data-limited model to be employed in interpreting our data. It attributes the precue effect to the greater "weight" given information from the precued element in choosing a response. (An alternative data-limited model, in which the observer separately decides whether each element is or is not a target, is discussed later in the paper.)

\section{WEIGHTED INFORMATION INTEGRATION}

The idea of information integration in detection was originally used to explain how subjects detected an auditory signal which could be at either of two frequencies (Green, 1958; Swets, Shipley, McKay, \& Green, 1959). Subjects were seen as integrating (summing) two impressions, one from each frequency channel, as if the stimulus was a two-element array where either element might be a signal (target) element. A signal is reported only if the integrated impression exceeds some decision criterion. This idea was generalized to explain set-size effects in search of multielement visual arrays, with each additional element adding noise to the integrated impression (see, e.g., Eriksen \& Spencer, 1969; Kinchla, 1969, 1974).

The idea of weighted information integration is an elaboration of the simpler unweighted, or equally weighted, process. It can be illustrated as follows. Suppose you were given advice, concerning the purchase of a house, by two friends who were real estate novices and a third friend who was a real estate expert. You might say you "gave more weight" to the expert's opinion in reaching a decision, or even that you "paid more attention" to the expert's opinion. Note that rather than implying any difference in the intelligibility or clarity of the three opinions (each might have been simply "buy" or "don't buy"), the term "attention" refers here to the extent to which each opinion controlled your decision. Many studies of human information integration have shown that we often combine information from several sources as a simple weighted sum. For example, one's rating of the overall "likeability" of someone described by a list of adjectives seems to be based on a weighted sum of the "likeability" 
values assigned to each adjective, where the weights represent the influence of adjectives at different positions within the list (see Anderson, 1991). However, variability in such ratings is normally treated as a form of error variance to be averaged out. In contrast, the model of visual search we consider next represents variance in rating responses as a major determinant of target detectability.

A computational (mathematical) model of visual search incorporating this idea was developed in earlier papers by Kinchla and Collyer (1974) and Kinchla (1974, 1977). It explains precue effects in terms of how much weight an observer assigns to his/her impressions of the individual elements when reaching a decision, with more weight assigned to the impressions of a precued element. Even if the quality (noise level) of each impression is the same, the more heavily weighted impressions of the precued elements have a greater influence on the observer's response. This leads to an increase in the detectability measure $d^{\prime}$ for targets in precued locations and a reduction in other locations.

Before briefly outlining the weighted integration model, it will be useful to introduce a more formal notation for describing the experimental task. Let $e_{i}$ denote the $i$ th element in an $n$-element stimulus array $(i=1,2, \ldots, n)$. Let $A_{0}$ denote an $n$-element array containing only nontarget elements and $A_{j}$ denote a similar array containing a single target element, $e_{j}(j=1,2, \ldots, n)$, such that, for example, $A_{4}$ denotes an array in which $e_{4}$ is a target and the other $n-1$ elements are nontargets.

\section{The Model}

1. Let each element $e_{i}$ evoke an elementary impression $X_{i}$, which is a Gaussian random variable (GRV) with variance $\alpha$ and an expected value of 1 if $e_{i}$ is a target or 0 if it isn't. ${ }^{3}$

2. Let the observer assign a nonnegative weight $\omega_{i}$ to each evoked value of $X_{i}$ to form an integrated impression, $Y$, where

$$
Y=\Sigma \omega_{i} X_{i}
$$

and $\Sigma \omega_{i}$ equals $1 .^{4}$

3. Let the observer report a target (a "yes" response) if $Y$ exceeds a response criterion, $C$, and otherwise report no target ("no").

\section{Properties of the Model}

It can easily be shown that the preceding assumptions (see Kinchla \& Collyer, 1974) lead to the familiar statistical decision problem illustrated in Figure 2. The integrated impression, $Y$, is a GRV whose expected value equals 0 given a nontarget array, $A_{0}$, and equals $\omega_{j}$ given a target array $A_{j}$ (an array in which the $j$ th element, $e_{j}$, is the single target element, $j=1,2, \ldots$, or $n$ ).

The variance of the integrated impression, $Y$, denoted $\sigma_{Y}^{2}$, depends only on the weights $\left(\omega_{i}\right)$ assigned to each of the $n$ array elements and on the noise parameter $\alpha$ (the variance of each elementary impression):

$$
\sigma_{Y}^{2}=\sum \omega_{i}^{2} \alpha
$$

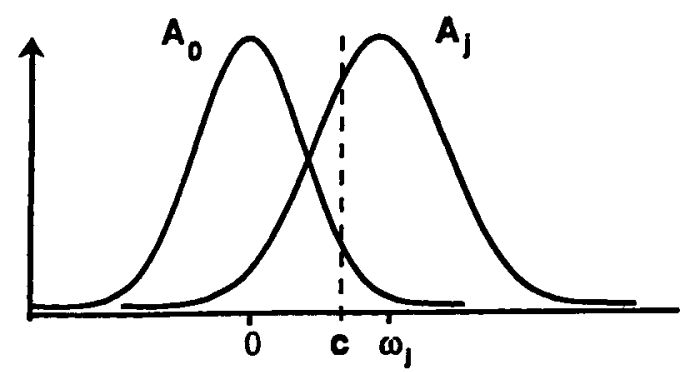

\section{Y: INTEGRATED IMPRESSION}

Figure 2. The integrated impression $Y$ is normally distributed with variance equal to $\Sigma \omega_{i}^{2} \alpha$ (see Equation 2), and an expected value of zero, given a nontarget array $\left(A_{0}\right)$, or $\omega_{j}$ (the weight assigned to the target element), given a target array $\left(A_{j}\right)$.

The observer's ability to discriminate one of the $A_{j}$ target arrays $\left(A_{1}, A_{2}, \ldots, A_{n}\right)$ from a nontarget array $\left(A_{0}\right)$ can be characterized by a $d^{\prime}$ detectability measure of the sort employed in the theory of signal detection (Green \& Swets, 1966). Specifically, $d_{j}$ is the difference between the expected value of $Y$ given an $A_{0}$ array (zero) and its expected value given $A_{j}\left(\omega_{j}\right)$, divided by the standard deviation of $Y$ (the square root of $\sigma_{Y}^{2}$ in Equation 2):

$$
d_{j}=\omega_{j} /\left[\Sigma \omega_{i}^{2} \alpha\right]^{1 / 2} .
$$

Note that $d_{j}$ ranges from 0 (when $\omega_{j}$ is 0 ) to a maximum of $1 / \alpha^{1 / 2}$ (when $\omega_{j}$ equals 1 ). Furthermore, $d_{j}$ becomes infinitely large as the elementary variance (noise) $\alpha$ approaches 0 , and approaches a limit of 0 as $\alpha$ becomes infinitely large.

The proportions of "yes" responses to each of the $n$ target arrays $\left(A_{1}, A_{2}, \ldots, A_{n}\right)$ represent $n$ hit rates, while the proportion of yes responses to an $A_{0}$ array represents the single false-alarm rate. Each $d_{j}$ can be estimated using this false-alarm rate and the appropriate hit rate to enter a conventional table of $d^{\prime}$ values (Green \& Swets, 1966). For example, the precue effects illustrated earlier in Figure 1 can be interpreted in terms of the weighted integration model, with the noise (variance) $\alpha$ of each elementary impression equal to .15 and the weight assigned to the precued element equal to .38 , assuming each of the other three elements had a weight equal to $(1-\omega) / 3$. The theoretical values of $d_{j}$ given these parameter values are indicated by the solid line in Figure 1. These results will be discussed again later in relation to the cue effects obtained in our own two experiments.

\section{EXPERIMENT 1}

Since precues are presented prior to both the subject's observation of an array and his/her decision concerning a response, they can influence both. However, a postcue presented well after both the array and a postarray mask but before the subject's response should influence only decision making. If such postcues have effects analogous to precues, it seems unlikely that the effects are due to a 
subject's allocation of some limited resource (attention) during the observation. Instead, it would seem to require an explanation in terms of the decision process, the sort of explanation afforded by the weighted integration model where postcues influence the integration weights $\left(\omega_{i}\right)$.

In order to assess the effect of such postcues, 3 observers participated in a visual search task involving four-element arrays with brief and simultaneous presentation of the four elements. A single target was present in a random two-thirds of the arrays and the rest were nontarget arrays. A postcue, presented after the array but before the response on each trial, indicated which of the four elements was most likely to have been a target. This cue was valid on a random two-thirds of the target trials and invalid on the rest. The visibility of the array elements was also varied by presenting them at two luminance ratios with their background. Thus there were two primary variables: the postcue, which should influence $\omega_{i}$ but not $\alpha$, and the luminance ratio, which should influence $\alpha$ but not $\omega_{i}$.

\section{Method}

Subjects. There were 3 paid female observers with normal or corrected-to-normal vision.

Apparatus and Stimuli. A Macintosh IIci computer with a 14in. RGB monitor was used to present stimuli and record responses. While seated in a dimly illuminated room, the observers viewed the monitor from a distance of approximately $118 \mathrm{~cm}$. A commercially available graphic program (Superpaint) and experimental program (VScope) were used to generate and display the stimuli.

Procedure. Observers first saw a fixation display consisting of four small, open squares arranged at the corners of a larger imaginary square centered on a fixation point. The side of each small square subtended $.73^{\circ}$ visual angle, and the side of the larger square subtended $1.7^{\circ}$. Two seconds after the fixation display came on, a four-letter array was presented for $15 \mathrm{msec}$ (one raster of the video display). Each letter appeared within (and almost filled) the area of one of the preceding small squares. On target trials one of the letters was the target letter $\mathrm{M}$ and the rest were nontarget Ws; on nontarget trials, all four letters were Ws.

One second after the letter array, a postcue and mask appeared for $2 \mathrm{sec}$. This consisted of four black masking squares positioned over the previous letters, with an outline around one of the squares (the postcue) indicating which of the preceding four letters was most likely to have been a target. Two seconds later, this display went off accompanied by a brief tone indicating that the observer had $3 \mathrm{sec}$ to indicate whether or not she believed the display had contained a target (by pressing one of two keys). A plus or minus then appeared on the screen for $.5 \mathrm{sec}$, indicating whether the response was correct $(+)$ or not $(-)$. Then, when ready, the observer initiated the next trial by pressing the space bar. The trials averaged about $7 \mathrm{sec}$ each.

The observer's ability to discern the target was also manipulated by varying the luminance ratio between the letters and the background; specifically, the letters were at a luminance of either $10.4 \mathrm{fL}$ or $11.5 \mathrm{fL}$ against a constant background luminance of $18.1 \mathrm{fL}$. In either case, a random two-thirds of the arrays contained a target letter $M$ and three Ws; the other one-third contained four Ws. When a target was presented, it was equally likely to occur in any of the four positions. The postcue occurred on every trial, and when a target occurred it was in the cued position on two-thirds of the trials.

After about 6 days, or 2,500 trials, of practice, each observer was tested for 15 daily 2 -h sessions with brief rest periods as needed (trials were observer paced). There was also a 15 -min rest period half way through each session. Each of these 15 sessions yielded 432 trials of data, or a total of 6,480 trials of data for each observer.

\section{Results}

A summary of each observer's performance is given in Table 1, which presents the proportion of trials on which each observer responded "yes" to target and to nontarget arrays, at each postcue and each luminance ratio.

To assess cue effects, the proportions of yes responses given a valid and an invalid cue were calculated for each observer (ignoring luminance). These proportions were, respectively, .866 and .852 for Observer M.K., .843 and .798 for Observer J.S., and .870 and .803 for $\mathrm{Ob}$ server M.G. While each observer responded yes more often when the signal element was cued, this cue effect was statistically significant only for Observers J.S. and M.G. $\left[\chi^{2}(1)=1.64, p<.20\right.$ for M.K.; $\chi^{2}(1)=12.35, p<$ .001 , for J.S.; $\chi^{2}(1)=17.11, p<.001$, for M.G.]. To assess luminance ratio effects, the proportion of correct responses on high- and low-ratio trials were calculated ignoring the cue. The proportion of correct on high- and low-clarity trials were, respectively, .662 and .637 for Observer M.K., .806 and .732 for Observer J.S., and .782 and .729 for Observer M.G. Again, while all 3 observers were correct more often given a high-clarity array, this effect was statistically significant only for Observers J.S. and M.G. $\left[\chi^{2}(1)=2.19, p<.20\right.$, for M.K.; $\chi^{2}(1)=55.86$, $p<.001$, for J.S.; and $\chi^{2}(1)=5.18, p<.05$, for M.G.].

\section{Theoretical Analysis and Discussion}

While only Observers J.S. and M.G. had statistically significant chi-squares for the effects of luminance ratio and precue, the model was fitted to the data from all 3 observers simply to show that nothing about M.K.'s data was inconsistent with the model.

Using the proportions of yes responses on valid and invalid cue trials as two "hit rates," and the proportion on nontarget trials as a common "false-alarm rate," conventional (see Green \& Swets, 1966) estimates of $d^{\prime}$ for valid and invalid cue trials were computed for each observer. These are presented as data points in Figure 3. The postcue effect is indicated by the higher sensitivity measure $\left(d^{\prime}\right)$ on valid cue trials.

Table 1

Proportion of "Yes" Responses for Each of the Six Types of Trials in Experiment 1

\begin{tabular}{clcc}
\hline & & \multicolumn{2}{c}{ Luminance } \\
\cline { 3 - 4 } Observer & \multicolumn{1}{c}{ Target } & $\begin{array}{c}\text { High Ratio } \\
p(\text { "yes") }\end{array}$ & $\begin{array}{c}\text { Low Ratio } \\
p \text { ("yes") }\end{array}$ \\
\hline M.K. & In cued position & .873 & .860 \\
& In uncued position & .863 & .841 \\
J.S. & No target & .753 & .795 \\
& In cued position & .890 & .795 \\
& In uncued position & .838 & .758 \\
M.G. & No target & .329 & .368 \\
& In cued position & .882 & .859 \\
& In uncued position & .819 & .787 \\
& No target & .378 & .483 \\
\hline
\end{tabular}


These effects can be interpreted in terms of the model by estimating the noise parameter for high- and lowluminance-ratio arrays, denoted $\alpha_{\mathrm{H}}$ and $\alpha_{\mathrm{L}}$, respectively, and the weight given to the cued element, denoted $\omega$, assuming that each of the other three noncued elements are given a weight equal to $(1-\omega) / 3$. These estimates of $\alpha_{H}$, $\alpha_{\mathrm{L}}$, and $\omega$ are presented in Table 2 . They were simply chosen to minimize the squared deviations of the $d^{\prime}$ values defined by the model (indicated by the lines in Figure 3 ) and those calculated directly from the data (the data points in Figure 3). Again, while more elaborate estimation techniques might be employed, they could only improve the already close fit between model and data apparent in Figure 3. Note that, for Observers J.S. and M.G., the luminance ratio manipulation produced shifts in detectability $\left(d^{\prime}\right)$ reflected in the different estimates of $\alpha_{\mathrm{H}}$ and $\alpha_{\mathrm{L}}$, while the difference in detectability due to cue validity $\left(d_{\mathrm{I}}\right.$ vs. $\left.d_{\mathrm{V}}\right)$ is reflected in the single estimate of $\omega$, which accounted for the precue effect in both luminance-ratio conditions. Even Observer M.K.'s data can be interpreted in this way. The model predicts progressively smaller differences between $d_{\mathrm{I}}$ and $d_{\mathrm{V}}$ as $\alpha$ is increased. The failure to find a statistically significant effect of cue validity for Observer M.K. can be attributed primarily to her very high noise levels $\left(\alpha_{\mathrm{H}}\right.$ and $\left.\alpha_{\mathrm{L}}\right)$, since her estimated $\omega$ value (.31) was virtually the same as that of $\mathrm{Ob}$ server J.S. (.28) and that of Observer M.G. (.30). While the absolute costs and benefits with postcues (Figure 3 ) are smaller than those with precues (Figure 1), the postcues had a substantial effect on performance, even though they would seem to have occurred too late to influence the allocation of attention during observation of the array. Postcue effects, as measured by our estimates of $\omega(.31, .28$, and .30$)$, were also substantial even though smaller than that for the precues (.38). Further experi-

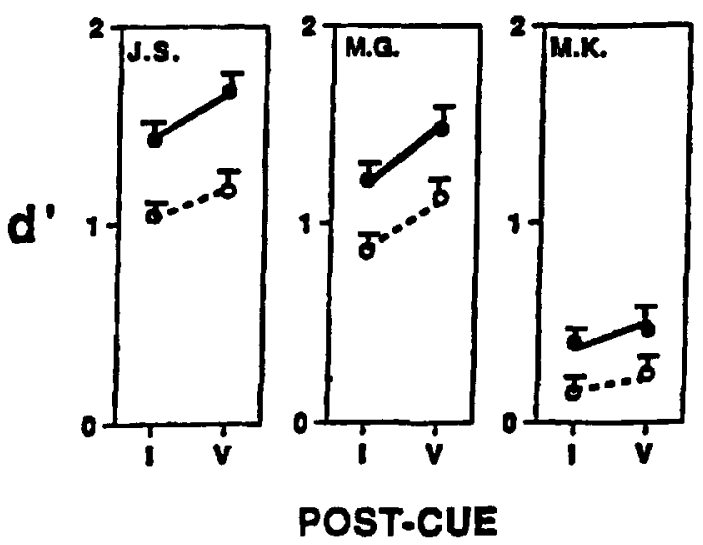

Figure 3. The four data points for each observer are estimates of $d_{j}$ for the high (solid points) and low (open points) luminance ratios given either an invalid (I) or valid (V) postcue. The bar on each point indicates the standard error of estimate based on the method described in Gourevitch and Galanter (1967). The lines indicate corresponding theoretical values based on Equation 3, using estimates of the noise levels for the high $\left(\alpha_{\mathrm{H}}\right)$ and low $\left(\alpha_{\mathrm{L}}\right)$ clarity conditions and a single estimate of $\omega$, the weight assigned to the precued element. These three estimates for each observer are shown in Table 2.
Table 2

Estimates for Each Observer of the Noise Parameters for High $\left(\alpha_{H}\right)$ and Low $\left(\alpha_{L}\right)$ Luminance-Ratio Conditions, and the Weight $(\omega)$ Given to the Cued Element, With Each of the Other Three Elements Given a Weight of $(1-\omega) / 3$

\begin{tabular}{cccc} 
& \multicolumn{3}{c}{ Parameter Estimates } \\
\cline { 2 - 4 } Observer & $\omega$ & $\alpha_{\mathrm{H}}$ & $\alpha_{\mathrm{L}}$ \\
\hline M.K. & .31 & 1.55 & 7.89 \\
J.S. & .28 & .111 & .218 \\
M.G. & .30 & .152 & .283 \\
\hline
\end{tabular}

Note--The theoretical values of $d^{\prime}$ in Figure 3 are based on these estimates.

mentation will be required to determine exactly why the postcue effects are somewhat smaller than Müller and Findlay's (1987) precue effects (Figure 1). For example, observers' coding strategies could be altered by the need to maintain representations of each element $\left(X_{i}\right)$ until the postcue is presented. Or the precue could influence both the noise level of the precued element and the weight assigned to it in the decision making, especially if the precue effects in Figure 1 are partially due to eye movement (see Note 2). In any case, a complete theory of visual search seems to require a data-limited explanation of our postcue effects.

\section{EXPERIMENT 2}

Our second experiment was designed to evaluate precue effects with sequentially presented array elements in order to compare them with those shown in Figure 1. If those effects are due to a greater share of some limited resource's being allocated to the precued element, sequential presentation should allow the entire resource to be allocated to each element in turn, producing different precue effects. However, if the precue effects in Figure 1 are simply due to more weight being given to the observer's impression of the precued element, then the precue effects with sequential presentation should be about the same as those with simultaneous presentation.

To assess precue effects with sequential presentation, 3 observers were shown a slow (one each $1.5 \mathrm{sec}$ ) succession of four briefly presented (about $45 \mathrm{msec}$ ) array elements on each of a series of trials. On each trial, they were to rate (on a 4-point scale) their confidence that the array contained a target letter, $M$, and three nontarget Ws, rather than four nontarget Ws. While observers knew that a random two-thirds of the arrays contained a target, the probability that a particular element was the target depended on which of two distribution conditions was in effect. In a uniform distribution condition, the observers were told that each element was equally likely to be a target, a neutral precue $(\mathrm{N})$. In a nonuniform distribution condition, the observers were told that the fourth element was seven times as likely to be a target as was each of the preceding three elements; thus this was a valid precue $(\mathrm{V})$ on a random $70 \%$ of such target trials and an invalid cue (I) on the other $30 \%$ of such trials. 


\section{Method}

Subjects. Three paid female observers with normal or correctedto-normal vision served as subjects.

Apparatus and Stimuli. The apparatus and commercially available software used to generate stimuli and record responses were the same as in Experiment 1, as were the observers' viewing conditions. Each array element was a dark blue circle $1.83^{\circ}$ in diameter, with an upper case letter, either $\mathrm{W}$ or $\mathrm{M}$, defined in a lighter blue, filling most of the circle. Each of the four successive elements was randomly, and independently, positioned within the confines of an imaginary $8^{\circ}$ square centered on a tiny $\left(.02^{\circ}\right)$ fixation dot permanently attached to the face of the CRT. The elements appeared against a $3.5-\mathrm{fL}$ white background. This value was selected to yield levels of accuracy comparable to those obtained by Müller and Findlay (1987) using simultaneously presented array elements (Figure 1).

Procedure. The observers were told to center their gaze on the fixation dot at the start of each trial. Then $(1.5 \mathrm{sec}$ after the offset of feedback for the preceding trial) four elements were successively flashed on for $45 \mathrm{msec}$ at the rate of one each $1.5 \mathrm{sec}$. The observers then had $1.5 \mathrm{sec}$ to press one of four keys to indicate their confidence that one of the preceding four elements was a target letter M. Immediately after each rating response, a feedback display was presented for $600 \mathrm{msec}$ : either the letter $\mathrm{M}$, indicating that the preceding array had contained a target, or a minus sign $(-)$, indicating that it had not. The first element presented on the next trial came on $1.5 \mathrm{sec}$ after the offset of this feedback. Each trial lasted a total of $10 \mathrm{sec}$.

Each observer performed 360 trials each day in two sessions of 180 trials, one session in the uniform distribution condition and the other in the nonuniform distribution condition, in counterbalanced order. The observers knew the condition in effect in each session. Each of the two daily sessions of trials took about $30 \mathrm{~min}$, and there was a $30-\mathrm{min}$ rest period between sessions.

Each observer was tested in this manner for about 4 days, until her performance appeared relatively stable, and then was tested for 8 days more to produce the 2,880 trials of data used in our analysis. These consisted of 960 target and 480 nontarget trials in each condition. The frequencies of the $A_{1}, A_{2}, A_{3}$, and $A_{4}$ arrays were, respectively, 240,240,240, and 240 in the uniform condition and $96,96,96$, and 672 in the nonuniform condition.

\section{Results}

Our first question was whether the 3 observers discriminated target from nontarget arrays at a better than chance level in both the uniform and nonuniform conditions. This was evaluated by treating ratings of 3 and 4 as "yes" responses and ratings of 1 and 2 as "no" responses. This allowed us to calculate the proportion of "correct" (yes to a target array or no to a nontarget array) responses for each observer in each condition. The proportions correct in the uniform and nonuniform conditions, respectively, were $66 \%$ and $65 \%$ for Observer Z.C., $73 \%$ and $71 \%$ for Observer L.S., and $81 \%$ and $79 \%$ for Observer H.Y. Chisquare tests comparing the tendencies to respond yes to target versus nontarget arrays for each observer in each condition indicated that each observer discriminated at better than chance levels in each condition $\left[\chi^{2}(1)=\right.$ $50,000, p<.001$, for each of the 3 observers]. We also determined that there was no significant difference in the pattern of responding to $A_{1}, A_{2}$, or $A_{3}$ arrays for each observer in either the uniform $\left[\chi^{2}(2)=1.28, p>.20\right.$, for Z.C.; $\chi^{2}(2)=4.64, p>.10$, for L.S.; $\chi^{2}(2)=3.15, p>.20$, for H.Y.] or the nonuniform $\left[\chi^{2}(2)=3.12, p>.20\right.$, for Z.C.; $\chi^{2}(2)=1.72, p>.20$, for L.S.; $\chi^{2}(2)=4.20, p>.10$, for H.Y.] condition. Of more central interest was each observer's pattern of responding to arrays containing a target in the first three positions $\left(A_{1}, A_{2}\right.$, or $\left.A_{3}\right)$ versus the pattern of responding to arrays containing a target in the fourth position $\left(A_{4}\right)$. A chi-square test based on the combined data for $A_{1}, A_{2}$, and $A_{3}$ arrays compared with $A_{4}$ arrays indicated a statistically significant difference in the pattern of responding for all 3 observers in the nonuniform condition $\left[\chi^{2}(1)=15.35, p<.001\right.$, for Z.C.; $\chi^{2}(1)=$ $50,000, p<.001$, for L.S.; $\chi^{2}(1)=15.37, p<.001$, for H.Y.], but no significant differences in the uniform condition $\left[\chi^{2}(1)=.12, p>.20\right.$, for Z.C.; $\chi^{2}(1)=1.23, p>.20$, for L.S.; $\chi^{2}(1)=1.56, p>.20$, for H.Y.]. In other words, all 3 observers responded similarly to a target in the first three positions or in the fourth position when targets were equally likely in each position (uniform distribution). However, their responses to targets in the first three positions were different from those to targets in the fourth position when a target was more likely to be in the fourth position (nonuniform distribution).

\section{Theoretical Analysis}

A theoretical interpretation of the data can be made in terms of the weighted integration model. Rating responses were organized to yield three points along a theoretical operating characteristic (OC) function, using the method described in Green and Swets (1966). One assumes that each observer selects three progressively larger values of the integrated impression, $Y$, dividing the abscissa in Figure 2 into four progressively higher regions, each eliciting a progressively higher confidence rating $(R=1,2,3$, or 4). One can then calculate three pairs of hit and falsealarm rates, the proportions $\hat{P}\left(R>V \mid A_{j}\right)$ and $\hat{P}\left(R>V \mid A_{0}\right)$, respectively, for $V=1,2$, and 3 . These define three progressively more conservative points along a theoretical OC specific to $d_{j}$ (Equation 3 ). Figure 4 presents data organized in this fashion for each observer in each condition. Each graph presents two sets of three points, one set based on the combined data from $A_{1}, A_{2}$, and $A_{3}$ trials (circular points) and the other on $A_{4}$ trials (triangular points). Since response tendencies were the same for a target in each of the four positions for each observer in the uniform condition, a single $O C$ was used to describe all six points for each observer. However, since response patterns for targets in the first three positions differed from those for targets in the fourth position in the nonuniform condition, two OCs were used to describe each observer's data. ${ }^{5}$

It is apparent in Figure 4 that the theoretical OCs are quite consistent with the data. While Observer H.Y. seems to have selected three rather similar criterion values, the data points for the other 2 observers spread well out along the OC functions. Note also that the OCs in the top row of graphs in Figure 4 indicate estimates of target detectability given a neutral precue, $d_{\mathrm{N}}$, and those in the bottom row detectability given a valid, $d_{\mathrm{V}}$, or invalid, $d_{\mathrm{I}}$, precue. These estimates are presented in the form of a cost-benefit analysis for each observer in Figure 5. The solid curves in Figure 5 indicate corresponding theoretical values based on the weighted integration model and 


\section{UNIFORM DISTRIBUTION CONDITION}

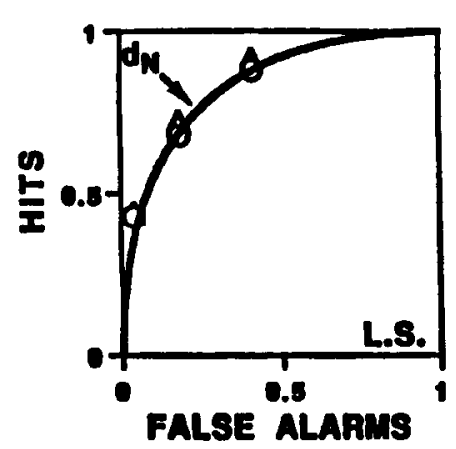

$O A_{1} A_{2} A_{3} \triangle A_{4}$
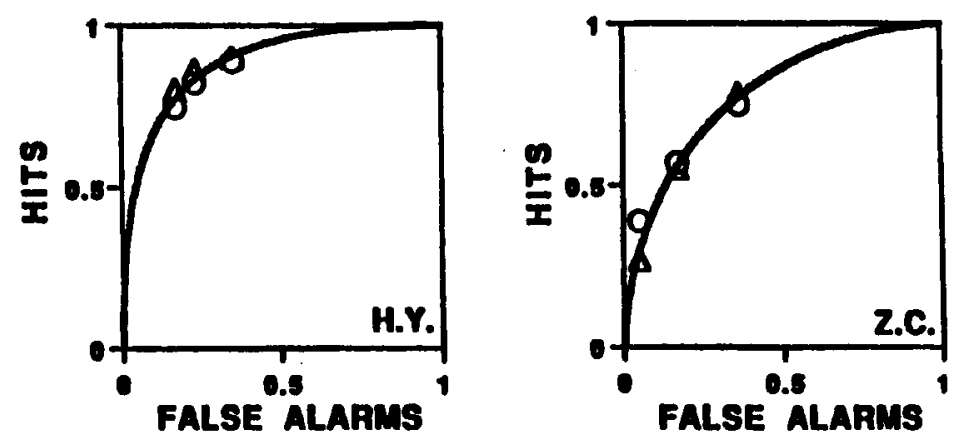

NONUNIFOAM DISTRIBUTION CONDITION
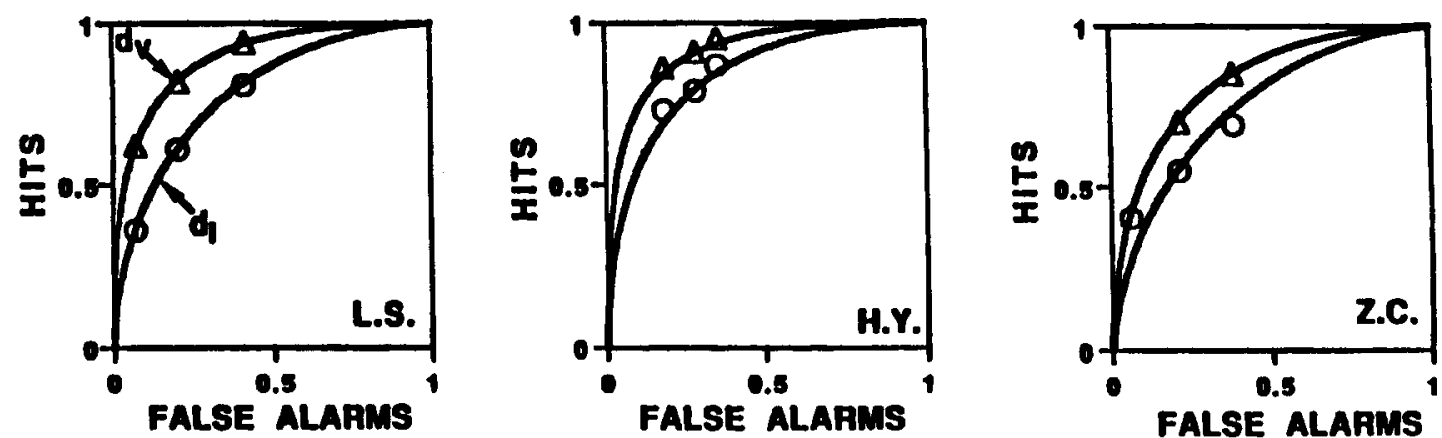

Figure 4. The rating data from Experiment 2 yields three points (see text) along an operating characteristic (OC) for targets presented in the first three intervals (circular points) and the last interval (triangular points) for each observer in each condition. A single $\mathrm{OC}\left(d_{j}\right)$ fits all six points in the uniform condition, whereas two $O C s$ are required to fit the six points in the nonuniform condition, with targets more detectable in the fourth interval (i.e., $d_{4}$ greater than $d_{1}, d_{2}$, and $d_{3}$ ).

estimates of two theoretical parameters: $\alpha$, the variance (noise) of each elementary impression, and $\omega$, the weight assigned to the precued element in the nonuniform condition, with each of the three less likely elements given weights of $(1-\omega) / 3$, since targets were equally likely to be detected in those three intervals. Each element in the uniform condition was assigned a weight of .25 , since our data indicated targets were equally detectable in all four intervals for each observer in that condition. The estimates of $\alpha$ and $\omega$ were chosen to minimize the squared discrepancies between the data points and the model's predictions.

Several aspects of Figure 5 are worth noting. Even though sequential presentation should eliminate the problem of sharing a limited resource among the four elements, the precue effects are of the same general magnitude as those obtained with simultaneous presentation (Figure 1). This similarity is consistent with the data-limited view of visual search represented in the weighted integration model, but difficult to explain in terms of a limitedresource view. Our model also suggests a way of distinguishing between precue effects and an observer's general level of sensitivity. Specifically, note that Observers H.Y., L.S., and Z.C. exhibit progressively lower levels of gen- eral sensitivity $\left(d^{\prime}\right)$ for each type of cue. This is reflected in the progressively higher estimates of the noise parameter ( $\alpha=.10, .14$, and .25, respectively). In contrast, the weight given to the precued element $(\omega)$ is identical for Observers L.S. and Z.C. (.34) and only slightly less for H.Y. (.31). It seems reasonable to consider $\omega$ as a measure of the precue effect and $\alpha$ as an inverse measure of general sensitivity (the lower the noise level $\alpha$, the more sensitive the observer). Thus, our 3 observers could be described as differing primarily in their general level of sensitivity $(\alpha)$ rather than the size of their precue effects $(\omega)$. For example, according to the model, simply increasing the luminance ratio of Z.C.'s letter elements until her noise level $(\alpha)$ is about .14 should cause her to perform just like Observer L.S.

Finally, it might be argued that "short-term memory" is a limiting cognitive resource when elements are presented sequentially. However, if this were the case, one would expect to find some sort of serial position effect in our data, whereas we found that targets were equally detectable (the pattern of rating responses was the same) in all four positions in the uniform condition and in the first three positions on the nonuniform condition. 

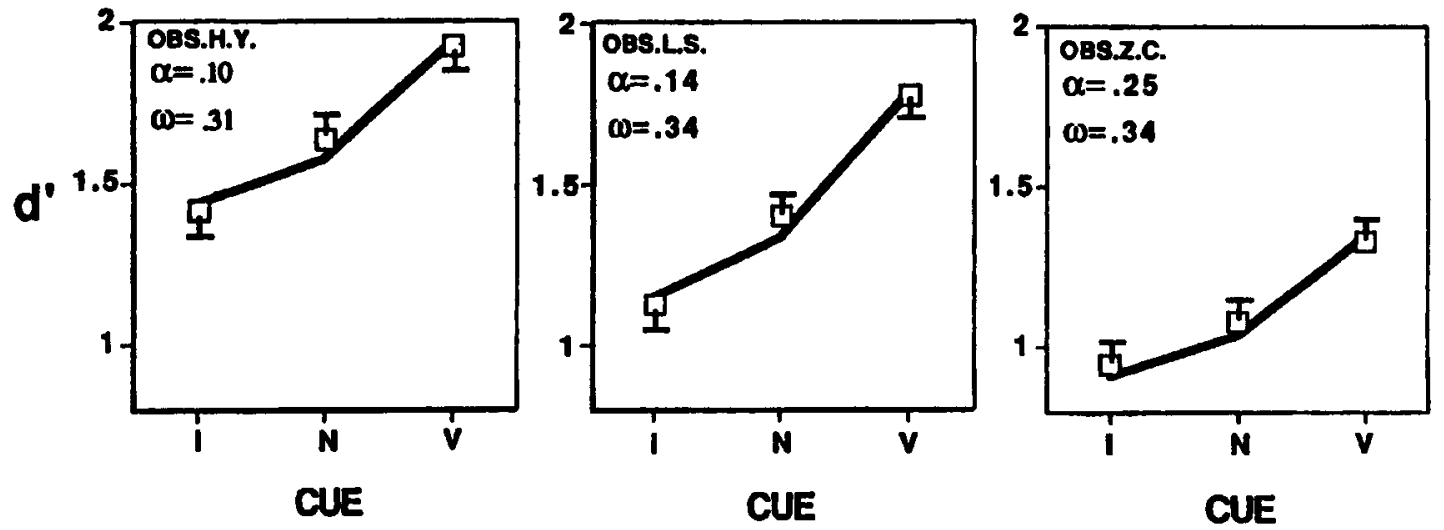

Figure 5. A cost-benefit analysis of each observer's data based on the operating characteristics shown in Figure 4. The bar on each estimate of $d^{\prime}$ indicates its standard error of estimate based on the method described in Gourevitch and Galanter (1967). Note that the progressively lower values of $d^{\prime}$ for Observers H.Y., L.S., and Z.C. can be attributed to their progressively lower general sensitivity to targets $(\hat{\alpha}=.10, .14$, and .25$)$. All 3 observers evidenced similar precue effects $(\hat{\omega}=.31, .34$, and .34$)$.

\section{GENERAL DISCUSSION}

While the postcue effects in Experiment 1 are smaller than the precue effects reported by Müller and Findlay (1987), they show that cues presented well after observation of an array can produce effects similar to those attributed to the allocation of "attention" during the observation. Experiment 2 showed that precue effects comparable to those reported by Müller and Findlay can be obtained with sequential presentation of array elements, which eliminates the problem of "sharing attention" among simultaneously presented elements, the limited resource explanation of precue effects. The data-limited view embodied in our weighted integration model provides an interpretation of precue effects consistent with both our experiments and that of Müller and Findlay. Thus, it represents a more parsimonious way of characterizing such effects than does the limited-resource view, which would require a totally different account of precue effects for simultaneous and sequential presentation.

The weighted integration model also provides a theoretical interpretation of cost-benefit effects which distinguishes between an observer's overall sensitivity $(\alpha)$ and the effect of the precue $(\omega)$. Our data (Figure 5) indicate that the smaller costs and benefits produced by lower sensitivity (larger $\alpha$ ) is independent of the precue effect $(\omega)$.

Our primary goal in this paper was to show how variation in weighting ( $\omega$ values) alone can account for certain cue effects. Thus one should not automatically attribute such effects to differences in the quality of information $(\alpha)$ extracted from each element due to a shift in attention. While our postcuing and sequential presentation paradigms are most easily interpreted as cue effects based on selective weighting, conventional precuing with simultaneous presentation of elements can be explained in terms of either $\alpha$ or $\omega$. Actually it seems quite likely (see Kinchla, 1992) that precuing an element in such tasks may produce both a clearer sensory representation of that element and increase its influence $(\omega)$ in decision making.
In any case, we have shown that our data-limited view by itself can account for many aspects of visual search currently attributed to the selective allocation of some limited processing resource.

\section{RELATED WORK}

Our experiments and our theoretical analysis of the data are relevant to a variety of other perceptual research.

\section{Weighted Integration and Reaction Time}

While the dependent variable in both of our experiments is accuracy, our results have implications for studies of visual search in which speed of responding is the principle dependent variable. A major issue in such studies is whether faster responding to precued elements represents more than a speed-accuracy tradeoff. Simply responding faster by sacrificing accuracy doesn't indicate any increase in sensitivity. However, an increase in both response speed and accuracy is usually taken as convincing evidence of heightened sensitivity. Our results imply that this conclusion may be incorrect. While development of this argument in terms of a formal model of reaction time is beyond the scope of this paper, the general idea is simple. Suppose an observer simply responds faster, thereby increasing the noise level $(\alpha)$ of every elementary impression. While this would reduce overall accuracy, an observer could compensate for the noisier representation of the precued element by assigning it more weight. Thus, an observer could be both faster and more accurate in detecting targets in precued locations, with no increase in sensitivity - that is, no change in the rate at which information is extracted from the stimulus array.

\section{Spatial Uncertainty and the Zoom-Lens Effect}

A number of studies have shown that the greater an observer's uncertainty regarding where in the visual field a target may occur, the harder it is to detect a target. This spatial uncertainty effect has led some to liken attention to a 
zoom lens which can pull back to cover a larger field or zoom in to cover a narrower one (e.g., Eriksen \& St. James, 1986). A limited resource view of attention would see this as spreading attention thinly over a wide area, or concentrating it on a precued location to enhance processing there. The weighted integration view provides an alternative explanation: an observer simply assigns more weight to impressions from the precued location in reaching a decision.

\section{Weighted-Integration and Guided Search}

Hoffman (1978, 1979), Egeth, Virzi, and Garbart (1984), and Wolfe, Cave, and Franzel (1989) have proposed limited-resource models in which the order of a serial search is influenced by some aspect of the array elements, such as color. For example, when searching an array for a red circle among green circles and red and green triangles, a subject may first identify red elements through a fast parallel process, then serially evaluate just those red elements for a circular one. This sort of process, which Wolfe et al. call guided search, could be more efficient than evaluating both red and green elements in a randomly ordered serial search. Color concentrates the successive allocation of "attention" (the limited resource) on elements that are most likely to be a target.

While the previously cited models of guided search account for a much wider range of phenomena (e.g., reaction-time data), the weighted integration model suggests an alternative conception. For example, suppose a subject is asked to search arrays of red and green triangles for a single, circular target element which may be either red or green, but is more likely red. Performance might be characterized by a weighted integration process similar to that defined earlier, but with weights assigned on the basis of an element's color rather than its position. Specifically, each element might evoke an elementary impression whose expected value is larger if that element is a circle rather than a triangle. The subject then integrates these impressions in a weighted manner, with higher weights assigned to red elements (since they are more likely to be target), reporting a circle present if the integrated impression exceeds some response criterion.

A fully developed illustration of a weighted form of guided search is presented in Kinchla (1974), where it is used to explain the role of structural redundancy in a visual search experiment. Subjects searched briefly presented pairs of large letters composed of small letters for a specific small target letter. If the target was more likely to occur in a specific large letter, subjects were better able to detect it in that large letter, as if the higher order form was guiding search for the constituent lower order target. This structural redundancy effect was shown to be consistent with a model much like the one used in this paper, with weights assigned to impressions of the small letters based on the large letter in which they occurred.

\section{Categorical Coding of Elementary Impressions}

In our model, subjects do not categorically code each elementary impression $X_{i}$ as a signal or nonsignal. Instead, they decide whether the integrated impression $Y$ is suffi- ciently strong to warrant an affirmative response. Other investigators (e.g., Shaw, 1982; Wickelgren, 1967) have proposed that subjects first decide whether each $X_{i}$ was evoked by a signal or a nonsignal, and respond affirmatively if even one $X_{i}$ is coded as a signal: specifically, $R_{1}$ occurs iff at least one $X_{l}$ exceeds its categorical coding criterion $C_{i}(i=1,2, \ldots, n)$. An independent decisions model of this sort can explain cue effects in terms of a cue's influence on the coding criteria $C_{i}$, with a more liberal (lower) criterion applied to the $X_{i}$ that a precue indicates is more likely to have been evoked by a signal. While Shaw (1982) does not explicitly consider precuing effects, she does evaluate two sets of letter-detection data to see whether our type of integration model or one in which the elementary impressions are categorically coded seems more appropriate. Unfortunately, Shaw's method of differentiating the two models is not feasible with our data, since it requires responses to all possible combinations of target and nontarget elements and only 5 of the $2^{4}$ such combinations occurred in our experiments. In any case, Shaw's results are equivocal. The integration model is clearly superior for one set of data and the categorical coding model is so for the other. Furthermore, some individuals in each study were consistent with each model. Such differences suggest that observers can adopt different coding strategies. Thus, postcuing ("probing") an observer to report whether a specific element was a target (e.g., Downing, 1988; Hawkins et al., 1990), or to follow a "target present" response by indicating exactly which element it was (e.g., Swensson \& Judy, 1981), may actually induce the observer to categorically code elementary impressions rather than integrate them in a weighted manner.

A closely related alternative to the independent decisions model is the extreme detector model in which only the largest or most signal-like impression, Xmax, is categorically coded: Specifically, detection occurs if $X \max$ exceeds the criterion $C$. (The independent decisions and extreme detector models are essentially equivalent when each of the $C_{i}$ in the former equal the single criterion $C$ in the latter.) The extreme detector model has considerable appeal, since it is closer to an optional decision process than to the simple integration process in our model, and leads naturally to the designation of $X \max$ as the single target element when the observer is asked to both detect (respond $R_{1}$ or $R_{0}$ ) and locate (indicate which one of the $n$ array elements is the target). It is clear that models of this sort can be elaborated to explain precue effects, either by assuming a more liberal criterion $\left(C_{i}\right)$ at a precued location or by defining $X \max$ as the maximum of weighted $X_{i}$ values, with the highest weight given to the precued element (Swensson, R. G., personal communication, 1994). Nevertheless our simple weighted integration model suffices here to illustrate how a purely datalimited process can account for precue effects without assuming any resource (attentional) limitation. For a fuller development of extreme detector models, the reader is referred to excellent papers by Graham, Kramer, and Yager (1987), Palmer, Ames, and Lindsey (1993), and Swensson and Judy (1981). 


\section{REFERENCES}

ANDERSON, N. (1991). Contributions to information integration theory. Hillsdale, NJ: Erlbaum.

Deutsch, J., \& Deutsch, D. (1963). Attention: Some theoretical considerations. Psychological Review, 70, 80-90.

Downing, C. G. (1988). Expectancy and visual spatial attention: Effects on perceptual quality. Journal of Experimental Psychology: Human Perception \& Performance, 14, 188-202.

Duncan, J. (1980). The locus of interference in the perception of simultaneous stimuli. Psychological Review, 87, 272-300.

EGETH, H. E., VIRZI, R. A., \& GARBART, A. (1984). Searching for conjunctively combined targets. Journal of Experimental Psychology: Human Perception \& Performance, 10, 32-39.

ERIKSEN, C. W., \& SPENCER, T. (1969). Rate of information processing in visual perception: Some results and methodological considerations. Journal of Experimental Psychology Monographs, 79(2, Pt. 2).

ERIKSEN, C. W., \& ST. JAMES, J. (1986). Visual attention within and around the field of focal attention: A zoom lens model. Perception \& Psychophysics, 40, 225-240.

ESTES, W. K., \& TAYLOR, H. A. (1964). A detection method and probabilistic models for assessing information processing from brief visual displays. Proceedings of the National Academy of Science, 52, 446-454.

Gourevitch, V., \& Galanter, E. (1967). A significance test for one parameter isosensitivity functions. Psychometrica, 32, 25-33.

Graham, N., Kramer, P., \& Yager, D. (1987). Signal-detection models for multi-dimensional stimuli: Probability distributions, and combination rules. Journal of Mathematical Psychology, 31, 366-409.

GreEN, D. M. (1958). Detection of multiple component signals in noise. Journal of the Acoustic Society of America, 30, 904-911.

GREEN, D. M., \& SWETS, J. A. (1966). Signal detection theory and psychophysics. New York: Wiley.

Hawkins, H. L., Hillyard, S. A., Luck, S. J., Mouloua, M., DownING, C. G., \& WoodwaRd, D. P. (1990). Visual attention modulates signal detection. Journal of Experimental Psychology: Human Perception \& Performance, 16, 802-811.

HoffMan, J. E. (1978). Search through a sequentially presented visual display. Perception \& Psychophysics, 23, 1-11.

HoFFMAN, J. E. (1979). A two-stage model of visual search. Perception \& Psychophysics, 25, 319-327.

KINCHLA, R. A. (1969). Temporal and channel uncertainty in detection: A multiple observation analysis. Perception \& Psychophysics, 5, 129-136.

KinCHLA, R. A. (1974). Detecting target elements in multi-element arrays: A confusability model. Perception \& Psychophysics, 15, 149-158.

KINCHLA, R. A. (1977). The role of structural redundancy in the perception of visual targets. Perception \& Psychophysics, 22, 19-30.

Kinchla, R. A. (1992). Attention. Annual Review of Psychology, 43, 711-742.

Kinchla, R. A., \& CollyeR, C. E. (1974). Detecting a target letter in briefly presented arrays: A confidence rating analysis in terms of a weighted additive effects model. Perception \& Psychophysics, 16, $117-122$.

KIEISS, J. A., \& LANE, D. M. (1986). Locus and persistence of capacity limitations in visual information processing. Journal of Experimental Psychology: Human Perception \& Performance, 12, 200-210.

MülleR, H. J., \& Findlay, J. M. (1987). Sensitivity and criterion effects in the spatial cuing of visual attention. Perception \& Psychophysics, 42, 383-399.

Norman, D. A., \& Bobrow, D. G. (1975). On data-limited and resource-limited processes. Cognitive Psychology, 7, 44-64.

Palmer, 3., Ames, C. T., \& Lindsey, D. T. (1993). Measuring the effect of attention on simple visual search. Journal of Experimental Psychology: Human Perception \& Performance, 19, 108-130.
PoSNER, M. I., \& SNYDER, C. R. R. (1975). Attention and cognitive control. In R. L. Solso (Ed.), Information processing and cognition: The Loyola Symposium (pp. 55-85). Hillsdale, NJ: Erlbaum.

Prinzmetal, W., \& Banks, W. P. (1983). Perceptual capacity limits in visual detection and search. Bulletin of the Psychonomic Society, 21, 263-266.

RUMELHART, D. E. (1970). A multicomponent theory of the perception of briefly exposed visual displays. Journal of Mathematical Psychology, 7, 191-218.

SHAw, M. L. (1982). Attending to multiple sources of information: I. The integration of information in decision making. Cognitive Psychology, 14, 353-409.

ShIFFrin, R. M., \& GARDNER, G. T. (1972). Visual processing capacity and attentional control. Journal of Experimental Psychology, 93, $72-83$.

SWENSSON, R. G., \& Judy, P. F. (1981). Detection of noisy visual targets: Models for the effects of spatial uncertainty and signal-tonoise ratio. Perception \& Psychophysics, 29, 521-534.

Swets, J. A., Shipley, E. F., McKey, M. J., \& Green, D. M. (1959). Multiple observations of signals in noise. Journal of the Acoustic Society of America, 31, 514-521.

Treisman, A. (1969). Strategies and models of selective attention. Psychological Review, 75, 282-299.

Treisman, A. M., \& Gelade, G. (1980). A feature integration theory of attention. Cognitive Psychology, 12, 97-136.

WiCKELGREN, W. A. (1967). Strength theories of disjunctive visual detection. Perception \& Psychophysics, 2, 331-337.

Wolfe, J. M., CaVe, K. R., \& Franzel, S. L. (1989). Guided search: An alternative to the modified feature integration model for visual search. Journal of Experimental Psychology: Human Perception \& Performance, 15, 419-433.

\section{NOTES}

1. A small increase in detectability with successively presented elements has been found under certain conditions (e.g., Duncan, 1980; Kleiss \& Lane, 1986; Prinzmetal \& Banks, 1983). However, none of these studies refute the conclusions shown by Eriksen and Spencer regarding the type of visual search tasks we consider in this paper.

2. In Appendix 3 of their paper, Müller and Findlay (1987) present two auxiliary experiments which they feel rule out any role of eye movements in their precue effects. While impressive, their arguments may not convince every reader, since eye movements were not monitored in the initial experiments and there were $750 \mathrm{msec}$ between the onset of the precue and the presentation of the test array.

3. Asserting that the expected value of $X_{i}$ equals 0 if $e_{i}$ is a nontarget and equals 1 if it is a target is an arbitrary choice of origin and unit which simplifies derivation. The predictions of the model remain the same so long as the expected value of $X_{i}$ when $e_{i}$ is a target is equal to or greater than what it is when $e_{i}$ is a nontarget.

4 . Asserting that $\Sigma \omega_{i}$ equals 1 is only a convenient way of representing $\omega_{i}$. The weights can be any set of nonnegative numbers, $\omega_{1}^{*}$, $\omega_{2}^{*}, \ldots, \omega_{n}^{*}$, since only their relative size is important in defining the $d_{j}$ values. For example, one can immediately transform each $\omega_{i}^{*}$ value to a corresponding $\omega_{i}$ value by simply dividing each $\omega_{i}^{*}$ by $\Sigma \omega_{i}^{*}$.

5. One OC was selected to pass through the circular point, and another $\mathrm{OC}$ to pass through the triangular point, for $V=2$ in the nonuniform-condition graphs in Figure 4. A single OC was selected to pass through the average of the corresponding two points in the uniformcondition graphs. While these are not maximum likelihood estimates of the theoretical OCs, this simpler estimation technique seems suitable here, since any more elaborate technique would only improve the already good fit between model and data.

(Manuscript received April 6, 1994; revision accepted for publication December $5,1994$. 